\title{
Anatomy of a wrong diagnosis: false Sinus Venosus Atrial Septal Defect
}

\author{
Nicola Gaibazzi*, Graziano Montresor and Maria Luisa Poeta
}

Address: Department of Cardiology, Ospedale Civile di Desenzano del Garda-Desenzano del Garda(BS), Italy

Email: Nicola Gaibazzi* - nicola.gaibazzi@inwind.it; Graziano Montresor - graziano.montresor@aod.it;

Maria Luisa Poeta - marialuisa.poeta@aod.it

* Corresponding author

Published: 07 November 2003

Cardiovascular Ultrasound 2003, I:15

This article is available from: http://www.cardiovascularultrasound.com/content/1/1/15

(C) 2003 Gaibazzi et al; licensee BioMed Central Ltd. This is an Open Access article: verbatim copying and redistribution of this article are permitted in all media for any purpose, provided this notice is preserved along with the article's original URL.
Received: 18 October 2003

Accepted: 07 November 2003

\begin{abstract}
In contrast with transthoracic echocardiography, transesophageal echocardiography provides a sure way to make the diagnosis of sinus venosus atrial septal defect; on the other hand this abnormality is more complex than that seen with the secundum atrial septal defect, and inexperienced operators may fail to recognize properly the defect. In front of a high reported sensitivity using transesophageal echocardiography, specificity is difficult to assess, due to possible underreporting of diagnostic errors. We describe a false positive diagnosis of sinus venosus atrial septal defect, in the setting of enlarged right chambers of the heart because of pressure overload. Modified anatomy of the heart, together with the presence of a prominent linear structure(probably Eustachian Valve) and an incomplete examination in this case made image interpretation very prone to misinterpretation. In this anatomical setting transesophageal longitudinal "bicaval" view may be sub-optimal for examining the atrial septum, potentially showing false images that need to be known for correct image interpretation. Nonetheless, a scan plane taken more accurately at the superior level would have demonstrated/excluded the pathognomonic feature of sinus venosus atrial septal defect in the high atrial septum, between the fatty limbus and the inferior aspect of the right pulmonary artery; moreover TEE allows morphological information about the posterior structures of the heart that need to be investigated in detail for a complete diagnosis.
\end{abstract}

\section{Background}

Transthoracic echocardiography has high sensitivity to detect secundum-type atrial septal defects(ASDs) and up to $100 \%$ for defects of the interatrial foramen primum, while its diagnostic usefulness for more uncommon causes of shunting at the atrial level is considerably less $[1,2]$. In particular, diagnostic images of the sinus venosus atrial septal defect (SVD) usually are not obtainable in most adults [1].
In contrast to clinical examination or transthoracic echocardiography, transesophageal echocardiography (TEE) provides a sure way to make the diagnosis of SVD; on the other hand this abnormality is more complex than that seen with the secundum ASDs, and inexperienced operators may fail to recognize the defect. TEE is a highly accurate means to diagnose SVD, especially when performed by experienced operators [3]. 

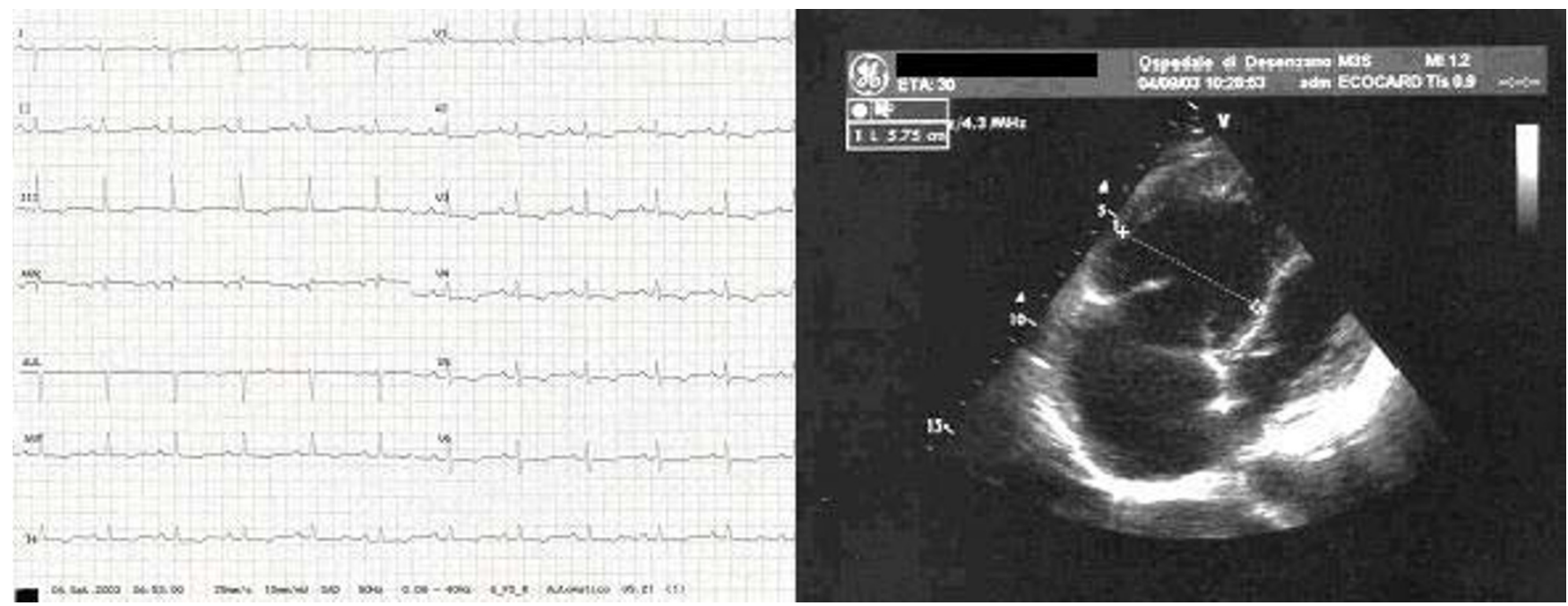

Figure I

ECG and transthoracic echocardiogram. ECG shows qR pattern in VI, QRS right axis deviation and diffuse repolarization abnormalities; transthoracic echocardiogram, apical 4-chamber view shows dilatation of the right atrium and of the right ventricle $($ max. diameter $=57 \mathrm{~mm})$.

In front of a high reported sensitivity for SVD diagnosis, specificity is difficult to assess, due to possible underreporting of diagnostic errors.

The following case report is to date the first in medical literature to describe a false positive diagnosis of SVD, even if probably it is not such a rare mistake, particularly in the setting of modified heart anatomy.

\section{Case report}

A 30-year-old woman presented for evaluation of multiple syncopal spells. She had been in her usual state of health until four months earlier when first syncopal episode presented; since then she has had five similar episodes. She was not using any type of medication; five years earlier she gave birth to a healthy baby, following an uncomplicated pregnancy. She described her syncopal episodes as typical for true syncope: episodes were 1) transient, 2) self-limited 3) leading to falling, 4) the onset was relatively rapid with spontaneous recovery.

ECG pattern was suggestive for right ventricle enlargement and strain (Fig 1).

Echocardiogram showed: massive dilatation of both the right atrium and the right ventricle, with a high estimated systolic pulmonary artery pressure $=75 \mathrm{mmHg}$. There was no clear evidence for any of the commonly diagnosed adult congenital abnormalities causing left to right shunt
(ASDs, ventricular septal defect, patent ductus arteriosus) (Fig 1).

CT scan of the chest, performed with the intent to rule out the presence of pulmonary thromboembolism was negative but confirmed right cavities enlargement with a consequential abnormal anatomical orientation of the heart; leftward shift of the atrial septum was evident too (Fig 2). A nuclear pulmonary perfusion scan was normal too, definitely excluding pulmonary thromboembolism. Transesophageal echocardiogram (TEE) showed the presence of a big ASD (maximum diameter $=29 \mathrm{~mm}$ ), (Fig 3). When agitated saline was injected from the right antecubital vein the air-bubbles showed to-and-fro shunting across SVD with changing atrial pressures during the cardiac cycle (Video 1-see additional file 1).

On the contrary cardiac cath ultimately demonstrated the absence of whatsoever ASD, confirming the presence of pulmonary hypertension, consequently diagnosed as primary.

We analyzed all the TEE images once again, trying to figure out where the diagnostic error originated; longitudinal "bicaval view" is usually utilized in TEE to investigate the atrial septum in its supero-posterior limbus, on a vertical plane starting from the inferior vena cava through the superior vena cava. In this case it is possible that this very view, usually ideal for most common secundum-ASDs 


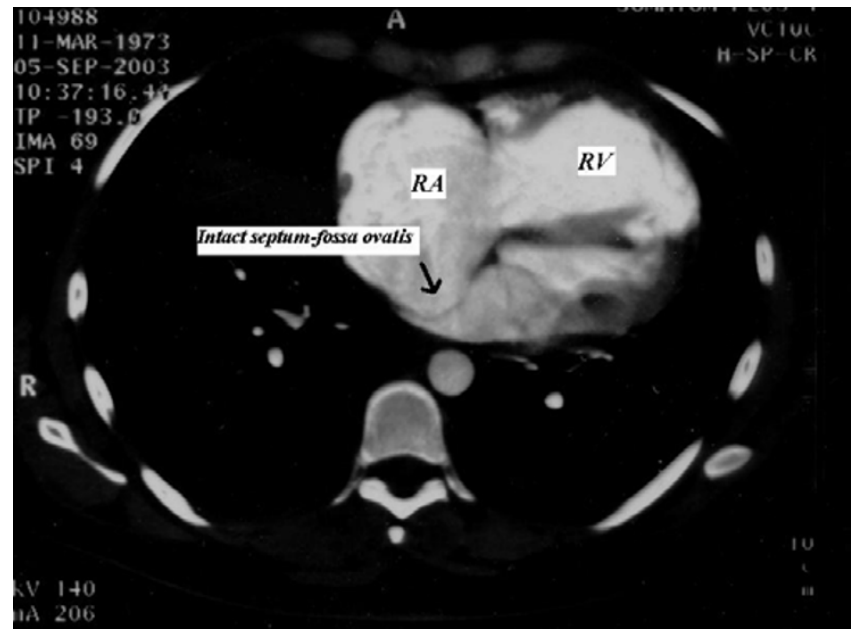

Figure 2

Contrast-enhanced chest CT scan. Four-chamber view of the heart. Right cavities enlargement with consequential abnormal anatomical orientation of the heart; leftward shift of the atrial septum is clearly shown.

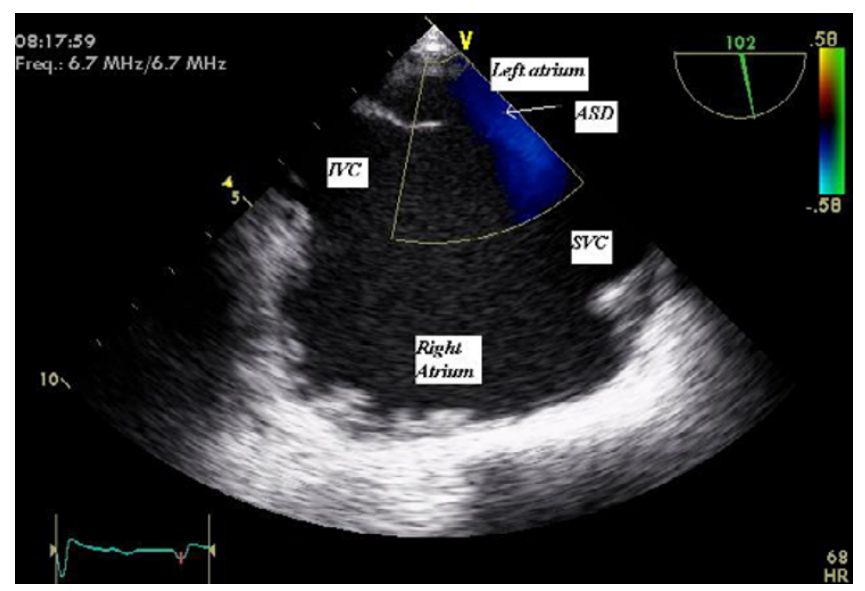

Figure 3

TEE-Midesophageal longitudinal view of both the atria and venae cavae. TEE shows the presence of a big ASD(maximum diameter $=29 \mathrm{~mm}$ ), SVD-superiorvena cava type. $R A=$ right atrium, LA=left atrium, IVC=inferior vena cava, SVC=superior vena cava.

detection, was misleading and inappropriately obtained so that high atrial septum was not shown.

In fact, getting back to the CT scan image, it appears that the abnormal heart morphology and orientation caused by right pressure overload, relatively to the normally posi-

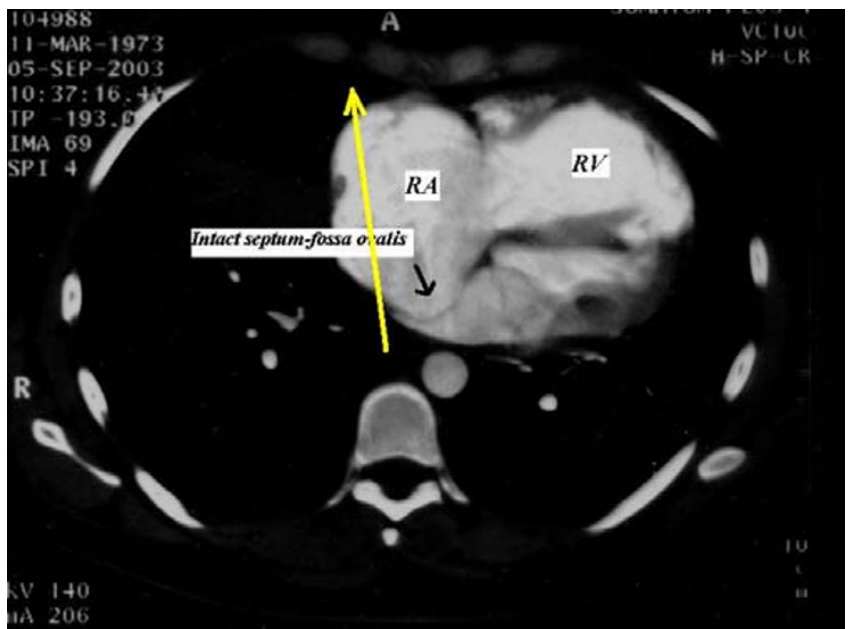

Figure 4

Same 4-chamber image as in Figure 2; the direction of the ultrasound beam, from the esophagus through the right atrium, is represented in yellow.

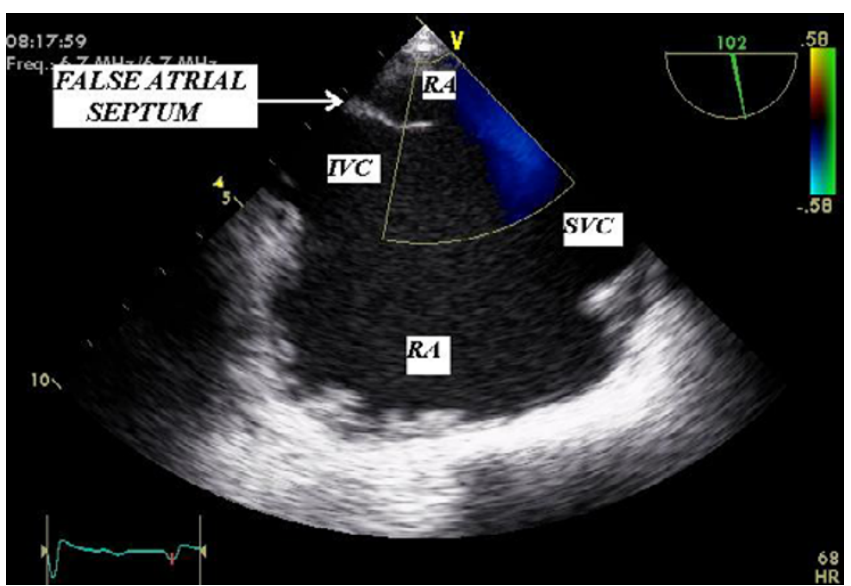

Figure 5

TEE-Bicaval longitudinal view. Same image as in Fig. 3 correctly reinterpreted. Image is reinterpreted in light of the atypical anatomy obtained by the cut-plane used, indicated by the yellow line in Fig. 4. Caption is consequently changed in the image. $R A=$ right atrium, IVC=inferior vena cava, SVC=superior vena cava.

tioned esophagus, modified the usual structures encountered by the ultrasound beam when oriented through both the venae cavae (these are used as a marker for correct longitudinal beam orientation); in this case, because of the massive right atrium dilatation, the beam was directed from the esophagus (not visualized) directly 


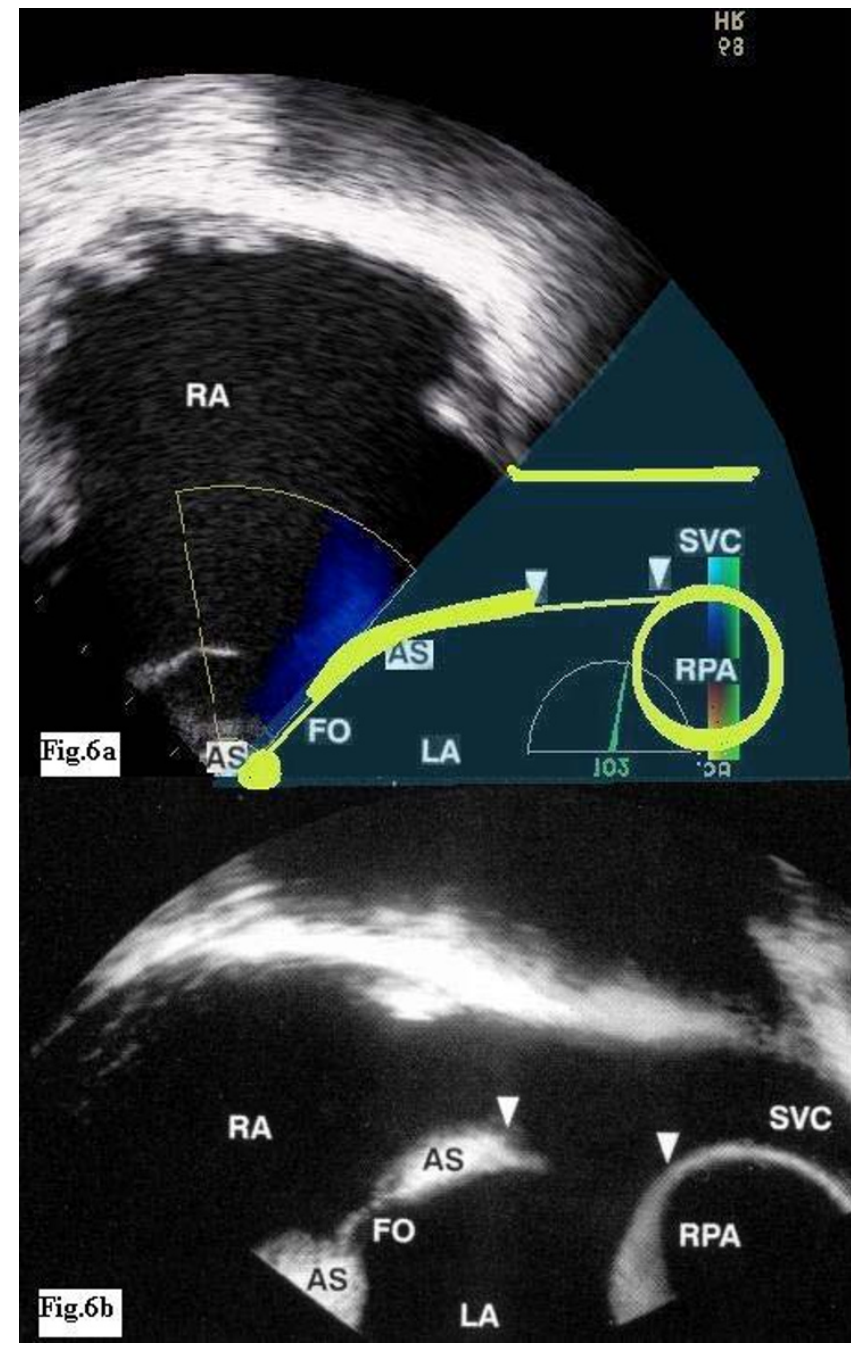

Figure 6

a) Same image as in Fig. 5, reoriented to be compared with Fig. 6b; the right part is added to show "virtual" anatomy(yellow line-drawing in the grey sector), should the probe be placed higher in the esophagus; b) Modified from"The echo manual $2^{\text {nd }}$ edition" by Oh JK, Seward JB, Tajik AJ. SVD image obtained using the correct scan plane(higher transducer positioning compared with Fig. 6a) for imaging of the higher atrial septum and eventual SVD; the presence of the right pulmonary artery(RPA), here showed in its short axis, posteriorly to the fatty limbus of the atrial septum, confirms correct positioning for eventual SVD imaging(SVD is definitely present in this case).

through the right atrium, "skipping" the two usually encountered structures in normal hearts, namely the left atrium and (even more importantly) the atrial septum (Fig. 4).
In this setting the presence of a linear echogenic structure, mistakenly interpreted as the atrial septum, apparently showed the absence of the superior limbus of the atrial septum, typical for SVD (Fig. 5). Very low patient compliance for probe in the high esophageal position limited the possibility to obtain potentially helpful images of the SVC-RA junction on the horizontal and longitudinal imaging-planes.

We can only speculate about the nature of this "false atrial septum" structure: it probably represents a prominent Eustachian Valve or it could be part of the Chiari network or it could simply be generated by right atrial extreme distortion and enlargement.

Nonetheless TEE examination was at best incomplete in this case, since we obtained only mid-atrial longitudinal images (fig. 3, fig. 5, Video 1-see additional file 1) not only inappropriate for SVD-Superior Vena Cava type diagnosing, but misleading in this particular anatomical setting.

This type of ASD can be correctly diagnosed only by a higher longitudinal scan with respect to the one we performed during the above-mentioned TEE exam.

A scan plane taken more accurately at a more superior level would have demonstrated the pathognomonic feature of SVD-Superior Vena Cava type, in the atrial septum between the fatty limbus and the inferior aspect of the right pulmonary artery (Fig. 6).

Moreover TEE has the potential to show morphological information about the posterior structures of the heart, that always need to be investigated in detail for a complete diagnosis since, in one third of the cases, SVD-Superior Vena Cava type is associated with anomalous entry of the right pulmonary veins to the heart.

\section{Conclusion}

a) An incomplete TEE exam, with no images of the most superior part of the atrial septum(between the fatty limbus and the inferior aspect of the right pulmonary artery) and b)the unlucky contextual presence of a prominent Eustachian Valve and a very dilated right heart, together were responsible for wrong image interpretation in this case; nonetheless careful evaluation of different views with different probe/beam orientation by a more experienced operator could have established the correct diagnosis.

Echocardiographers performing TEE should be aware of this pitfall when examining patients with enlarged right chambers and abnormal heart orientation; this may be more relevant for "real-world" adult echocardiographers, 
generally poorly trained in recognition of the rarest congenital abnormalities.

Mid-atrial longitudinal "bicaval" view, normally utilized to diagnose most common ASDs, is sub-optimal for SVDSuperior Vena Cava type; it is not only insufficient for a complete examination of the higher atrial septum, but, particularly when confronted with modified heart anatomy, it may potentially show false images that need to be taken into consideration for correct image interpretation.

\section{Additional material}

\section{Additional File 1}

Video1 TEE-Agitated saline contrast injection from right antecubital veinDouble click to start.

Click here for file

[http://www.biomedcentral.com/content/supplementary/1476-

7120-1-15-S1.wmv]

\section{References}

I. Shub C, Dimopoulos IN, Seward JB, Callahan JA, Tancredi RG, Schattenberg TT, Reeder GS, Hagler DJ and Tajik AJ: Sensitivity of twodimensional echocardiography in the direct visualization of atrial septal defect utilizing the subcostal approach: experience with I 54 patients. J Am Coll Cardiol I983, 2:127-I35.

2. Khandheria BK, Shub C, Tajik AJ, Taylor CL, Hagler DJ and Seward JB: Utility of color flow imaging for visualizing shunt flow in atrial septal defect. Int J Cardiol I989, 23:91-98.

3. Pascoe RD, Oh JK, Warnes CA, Danielson GK, Tajik AJ and Seward JB: Diagnosis of Sinus Venosus Atrial Septal Defect With Transesophageal Echocardiography. Circulation 1996, 94:1049-1055.
Publish with Bio Med Central and every scientist can read your work free of charge

"BioMed Central will be the most significant development for disseminating the results of biomedical research in our lifetime. "

Sir Paul Nurse, Cancer Research UK

Your research papers will be:

- available free of charge to the entire biomedical community

- peer reviewed and published immediately upon acceptance

- cited in PubMed and archived on PubMed Central

- yours - you keep the copyright

Submit your manuscript here:

http://www.biomedcentral.com/info/publishing_adv.asp 